\title{
Practice-Based Research Network Partnership with CDC to Acquire Clinical Specimens to Study the Etiology of Southern Tick-Associated Rash Illness (STARI)
}

\author{
Meagan F. Vaughn, MS, Philip D. Sloane, MD, MPH, Kyle Knierim, MD, Dax Varkey, \\ Mark A. Pilgard, and Barbara J. B. Johnson, PhD
}

Introduction: Erythema migrans (EM) is an annular, erythematous, expanding rash that is characteristic of early Lyme disease. In the southern United States, however, many cases of EM seem to have an etiology different from that of Lyme disease. This little-understood condition is called Southern tickassociated rash illness.

Methods: With the goal of obtaining biological specimens and clinical histories from 12 to 20 STARI patients for use in etiologic research, microbiologists from the Centers for Disease Control and Prevention contacted the North Carolina Network Consortium, a statewide consortium of practice-based research networks. This article describes the methods by which the North Carolina Network Consortium successfully identified and enrolled Southern tick-associated rash illness patients into a primary carebased research protocol.

Results: A total of 23 patients were enrolled, with $100 \%$ attainment of the desired specimens. After an initial lack of success, the revised protocol identified and trained physicians practicing in endemic areas for the illness, used a coordinator with 24-hour availability, recruited participants using newspaper notices and medical providers, and provided regular reminders and progress updates.

Conclusions: A practice-based research network can help basic scientists identify patients and collect specimens for clinically relevant research. (J Am Board Fam Med 2010;23:720-727.)

Keywords: Practice-Based Research Networks, PBRNs, Centers for Disease Control and Prevention, CDC, Southern Tick-Associated Rash

Erythema migrans (EM) is an annular, erythematous, expanding rash that is characteristic of early Lyme disease, an infectious disease that is endemic

This article was externally peer reviewed.

Submitted 22 April 2010; revised 8 July 2010; accepted 12 July 2010.

From the Department of Epidemiology, School of Public Health (MFV), the Department of Family Medicine, School of Medicine (PDS), and the Cecil G. Sheps Center for Health Services Research (PDS, KK, DV), University of North CarolinaChapel Hill, Chapel Hill; and the Centers for Disease Control and Prevention, Ft. Collins, CO (MAP, BJBJ).

Funding: Funding for this study was provided by contract no. 200-2008-M-24740 from the US Centers for Disease Control and Prevention, and by a supplemental contract from the North Carolina State Department of Health.

Prior presentation: Portions of this manuscript were presented at the 2009 National Practice-Based Research Network Conference, Bethesda, MD, June 22-24, 2010.

Conflict of interest: none declared.

Corresponding author: Philip D. Sloane, MD, MPH, Department of Family Medicine, University of North Carolina School of Medicine, CB 7595 Aycock Building, Chapel Hill, NC 27599-7595 (E-mail: psloane@med.unc.edu). in the northeast, north central, and Pacific northwest of the United States. In the southeastern and southcentral states, cases of EM have also been reported since the mid $1980 \mathrm{~s}^{1-3}$; however, antibodies to Borrelia burgdorferi, the cause of Lyme disease, are infrequently identified in these patients and no cultures of B. burgdorferi have been grown from them. ${ }^{1,2,4}$ Thus, EM in this region is believed to often represent an illness that is not Lyme disease, the etiology of which is unknown. This illness has been provisionally named Southern tick-associated rash illness (STARI).

Compared with Lyme disease, which is transmitted by the tick vector Ixodes scapularis, STARI is associated with the bite from the lone star tick (Amblyomma americanum) ${ }^{5-7}$ In recent years, $A$. americanum has been increasingly reported in areas endemic for Lyme disease. ${ }^{6,8}$ Because a clinical diagnosis of early Lyme disease is often based on 
the presence of an EM rash, the increasing area of overlap where both tick vectors are present creates difficulties for diagnosis and treatment of patients with EM in these regions.

Other than the known tick association, the etiology, natural history, and appropriate treatment of STARI remain largely unknown. Wormser et al ${ }^{9,10}$ compared microbiologic and clinical characteristics of patients who had EM rashes in New York (an area endemic for Lyme disease) and Missouri (a nonendemic area). Although $75 \%$ of patients from New York were seropositive for antibodies to $B$. burgdorferi, all patents with EM from Missouri were seronegative. The EM rashes, although generally similar in appearance, were slightly smaller, more circular, and more likely to have central clearing in Missouri patients than New York patients. In addition, Missouri patients had a milder illness with a shorter duration and fewer symptoms. ${ }^{10}$

Several studies have investigated the role of Borrelia lonestari and Rickettsia amblyommii as possible etiologic agents of STARI, but the results have indicated that they are unlikely to be the causal agents. ${ }^{9,11,12}$ Without an understanding of the etiology of STARI, development of clinical and surveillance case definitions and laboratory methods to differentiate STARI from early Lyme disease is not possible. Because the etiology of STARI remains unknown it is difficult to develop evidence-based treatment guidelines for STARI patients. For these reasons it is imperative that the etiology of STARI be determined.

Difficulties with recruiting patients who have STARI and obtaining suitable biological specimens and clinical data have been limiting factors in the research efforts toward identifying the etiology of STARI. Convalescent serum samples, which require the patient to return for a second visit several weeks after the initial visit, can be particularly difficult to obtain. Because it is likely that the majority of STARI patients present to primary care physicians, the primary care office is a logical setting in which to seek to obtain biological specimens for research. However, practice-based research studies tend to suffer from problems with recruitment and retention of primary care physicians because of such issues as a lack of interest in the research topic, the requirement of a large time commitment, poor compensation, not having a voice in the research process, and not seeing the results of experimental tests as they are performed. ${ }^{13,14}$
Practice-based research networks (PBRNs) have become an important mechanism to involve primary care physicians in clinical and epidemiological research. In this article we describe a partnership between microbiologists at the US Centers for Disease Control and Prevention (CDC) and a statewide PBRN for the purpose of obtaining patient specimens to help identify the etiologic agent of STARI. We describe the methods used to engage primary care physicians in this basic science research and to complete a complex data collection protocol.

\section{Methods}

In 2006 the North Carolina Family Medicine Research Network was contacted by the CDC and the North Carolina state epidemiologist for assistance in identifying physicians to participate in a research protocol to collect clinical information and biological specimens from patients who had STARI. At the time, the CDC had a multistate protocol in place that allowed physicians to submit serum, blood, and skin biopsy samples from patients who had STARI to the CDC, but had limited success in enrolling STARI patients.

\section{Data Collection Sites}

To identify a geographic region with a high probability of finding cases of STARI, we surveyed providers at 5 practices: 3 in eastern and 2 in central North Carolina. Practices were selected using a convenience sample from regions that, according to state epidemiologic data, had frequently reported cases of EM in previous years. After observing a presentation about STARI, providers in each practice were surveyed regarding the number of cases they had seen in the previous year and whether they would be interested in participating in a research protocol. The results of the preliminary survey are shown in Table 1. Among the 5 practices, only those in eastern North Carolina reported an average of greater than one STARI case per provider in 2007.

Based on the results of the survey we decided to focus our data collection efforts in eastern North Carolina. In addition to the 3 practices included in the survey, we also recruited a fourth practice in this region. In each practice, one provider was identified to act as site coordinator. Pamphlets containing information about the study and photo- 


\begin{tabular}{lcccc}
\hline & & Providers Interested \\
Practice & Providers Surveyed (n) & $\begin{array}{c}\text { Provider Estimates of STARI Cases Seen during } \\
\text { 2007 } \\
\text { the Study (n) }\end{array}$ & $\begin{array}{c}\text { Cases Seen by Practice } \\
\text { (Total n) }\end{array}$ & $\begin{array}{c}\text { Cases Per Provider } \\
\text { (Mean) }\end{array}$ \\
\hline Eastern NC 1 & 3 & 2 & 6 & 2.0 \\
Eastern NC 2 & 4 & 4 & 12 & 3.0 \\
Eastern NC 3 & 3 & 3 & 37 & 12.3 \\
Central NC 1 & 29 & 21 & 5 & 0.17 \\
Central NC 2 & 6 & 4 & 6 & 1.0 \\
\hline
\end{tabular}

STARI, Southern tick-associated rash illness; NC, North Carolina.

graphs of STARI rashes were placed in patient waiting rooms and handed out to all nurses and physicians in each practice. All other providers within each practice were asked to refer all STARI patients to the site coordinator for study recruitment and enrollment. The site coordinators attended training to learn the study protocol and to practice data collection and collection of skin biopsy specimens. The training session included a detailed discussion and review of the study protocol and study forms. All physicians were also required to practice performing data collection using the actual forms and kits to be used in the study. Skin punch biopsy technique was reviewed using pig feet as specimens. In addition, weekly emails were sent to all site coordinators to update them on the progress of the study and to encourage continued participation. All project staff and physicians were given access to a secure Internet site (http://blackboard.unc.edu), which was created specifically for the study and housed electronic copies of all study protocols and documents. The study protocol was approved by the institutional review boards for the protection of human research subjects at the CDC, the University of North Carolina (UNC), and Eastern Carolina University. Study physicians completed human research ethics training and were approved under either the UNC or Eastern Carolina University institutional review boards.

After the first month of the study (May 2008), which was reportedly the start of the peak of "tick season" for A. americanum, ${ }^{15}$ no patients had been enrolled. Therefore, 3 modifications were made: (1) a new site was recruited in central North Carolina-a large academic site in which a junior faculty member agreed to coordinate data collection; (2) notices about STARI and about the study were placed in local newspapers in the areas where the practices were located; and (3) a universitybased student research assistant began carrying a mobile phone so as to be available to screen respondents to the newspaper notices and to answer questions from the study physicians. Patients who were recruited through the newspaper advertisements were referred to the designated on-call site coordinator or the site coordinator who was located nearest to the patient's residence. This phase of the study was conducted from May 1 through August 31,2008 , after which the study was discontinued for the year because the season of greatest risk of lonestar tick bites had ended in the study communities.

A second period of patient recruitment was organized the following spring. This time only sites in central North Carolina were included because the majority of subjects in year 1 had been recruited in that region. Three new study physicians were recruited (the site coordinator from the year before, who had enrolled 7 of the 10 subjects, was not available). All 3 physicians had practices based in central North Carolina and had expressed interest in STARI and other tick-borne diseases. The provider training session and start of patient recruitment took place in early April 2009. Patients were enrolled from the middle of April until June 1, 2009.

\section{Patient Recruitment Criteria}

The eligibility criteria included age 18 years or older; the ability to provide written consent; an annular, erythematous, expanding EM-like rash that started within 2 weeks (2008) or 4 weeks (2009) of the enrollment visit and that was at least $5 \mathrm{~cm}$ in diameter; a history of tick bite at the rash site or potential exposure to ticks within 2 weeks (2008) or 
4 weeks (2009) before onset of the rash (differing time frames for onset of the rash and tick bite history were the result of a requested change in the protocol by the CDC to reflect evolving case definitions); no alternative explanation for the rash; consent to providing skin biopsies during the initial visit and blood samples at both the initial and follow-up visits; and consent to storage of biologic samples for later testing. Physicians were instructed to exclude patients who met any of these criteria: currently taking antibiotics; rash location on the face, neck, or genitals; hemophilia or other coagulopathy or taking warfarin; immunocompromised; a history of or risk factors for a severe skin or wound infection; a history of keloid formation; pregnancy; or any unstable medical condition. $\mathrm{Pa}-$ tients who were eligible for the study and who expressed interest in participating were asked to complete the informed consent process.

\section{Data and Specimen Collection Procedures}

Once a potential study participant was identified, the site coordinator conducted a preliminary interview to determine whether study eligibility criteria were fulfilled. If so, the site coordinator arranged to see the patient within 24 hours to ensure timely collection of clinical specimens. Because STARI rashes may fade quickly, it was imperative that the samples be collected as soon as possible after the onset of the rash and before taking antibiotics to increase the likelihood of finding any infectious agent(s) that might play a role in the etiology of STARI.

Physicians completed a patient interview and physical examination, recording clinical and tick exposure information. Several photographs of the rash were taken to document its size, shape, and coloring. The biologic specimens collected from the patient during the first visit included serum, anticoagulated whole blood, and 2, 2-mm skin punch biopsies. When available, the tick that was attached to the bite site, either removed by the physician or brought in by the patient, was collected and submitted to the CDC along with the patient specimens. Patients were provided with wound care instructions to prevent infection of the skin biopsy sites.

All patients were asked to return for a follow-up visit 3 to 6 weeks after the first visit. During this visit a patient interview and physical examination were completed and a convalescent serum sample was collected. Patients were reimbursed \$200 for the first study visit and $\$ 100$ for the follow-up study visit. Physicians were reimbursed $\$ 1000$ per patient who was enrolled in the study. Inclusion of a protocol checklist (Figure 1) in the packet of study forms in each specimen collection kit proved to be a useful strategy for ensuring completion of all required components (including informed consent, patient and provider compensation forms, data collection forms, and specimen collection). Samples, data collection forms, and a copy of the consent form were shipped overnight to the CDC laboratory in Fort Collins, CO. Copies of all study forms were also mailed to the study coordinators at UNC, who were responsible for patient tracking and disbursement of patient and physician incentives.

The CDC was an active partner in the project. A CDC scientist attended both data collector training sessions, lending expertise and emphasizing the importance of the project. During data collection, CDC staff conducted follow-up calls or E-mails with the study physicians after each patient was enrolled, fielded questions from physicians, and sent new specimen collection kits as needed. One patient contacted the CDC directly and was referred to one of the site coordinators for enrollment into the study. Once the samples were received by the CDC they were processed, tested, and stored for further serologic and molecular analyses.

\section{Results}

In 2008, 10 patients were successfully enrolled during 16 provider-months of data collection; of these, 7 were recruited by the new study physician in central North Carolina. In 2009, 13 patients were successfully enrolled during 4.5 provider-months of data collection, for a final total of 23 study participants (Table 2). The majority of the study participants were identified and recruited directly by the site coordinators from their own patients or through referral from another physician within their practice.

The newspaper notices generated a large response from the general community. Of the 28 calls received in April and May of 2009, most were patients who had a small localized reaction to a recent tick bite that was not large enough to qualify them for the study (and which is a common reaction to irritating tick saliva), or were persons who 
Figure 1. Protocol checklist used by study physicians for patient enrollment and data collection.

\section{UNC STARI Study}

\section{Protocol Check List}

Confirm that the patient is interested in participating in the study.

Confirm that the patient passes the eligibility \& exclusion criteria:

\section{Eligibility Criteria}

- At least 18 years old

- Able to provide written consent

- Annular, erythematous, expanding EM-like rash that began within 4 weeks of the visit to provider's office and is at least $5 \mathrm{~cm}$ in diameter

- History of tick bite at the rash site OR potential exposure to ticks within 4 weeks before rash onset

- No alternative explanation for the rash

- Will consent to skin biopsy and blood sample now and blood sample 3-6 weeks later

- Will consent to storage of biologic samples for later testing

\section{Exclusion Criteria}

- Currently taking antibiotics

- The EM rash is on the face, neck or genitals

- Has hemophilia or other coagulopathies, or taking warfarin (Coumadin)

- Currently undergoing chemotherapy or otherwise severely immunocompromised

- Past history of, current problem with, or anticipated severe skin or wound infections.

- History of keloid formation

- Pregnant

- Non-English speaking (unless you are fluent in his/her language)

- Medically unstable (at discretion of provider)

Proceed through informed consent process. Have patient sign three (3) copies of the consent form (one each for UNC, CDC, and your practice's records) and give him/her a fourth, blank copy to retain.

Have patient sign HIPAA Authorization and give him/her a blank copy to retain.

- Have the patient sign Social Security Number Disclosure Addendum and Patient Reimbursement Form.

$\square$ Assign a unique Subject Record Number. Mark it on the Subject Tracking Form.

$\square$ Complete the Laboratory Submission Form for Southern Tick-Associated Rash Illness (STARI) Specimens. Make a copy of this form to be mailed to UNC. The original will be mailed to the CDC.

$\square$ Photograph the rash with ruler (labeled with subject ID sticker) in the frame. Mark photo numbers on Subject Tracking Form.

$\square$ Collect (2) skin biopsies, placing them in separate saline tubes included in the kit, and refrigerating them.

$\square \quad$ Collect Unclotted (purple topped tube) and Clotted (red/grey topped tube) serum samples.

$\square \quad$ Label all specimen tubes with Subject Record Number using a permanent marker

$\square \quad$ Schedule a follow-up appointment. Mark it on the Subject Tracking Form along with contact method to remind him/her of the appointment.

$\square$ Ship to CDC:

\begin{tabular}{|c|c|c|}
\hline $\begin{array}{l}\square \\
\square \\
\square \\
\square \\
\square\end{array}$ & $\begin{array}{l}\text { Itemized list of contents } \\
1 \text { st Signed Informed Consent Form } \\
\text { Laboratory Submission Form } \\
\text { All blood and tissue specimens with gel-type ice packs } \\
\text { Tick (if applicable) }\end{array}$ & $\begin{array}{l}\text { Ship to: } \\
\text { CDC Foothills Campus } \\
\text { Bacterial Diseases Branch } \\
3150 \text { Rampart Road } \\
\text { Fort Collins, CO } 80521 \\
\text { ATTN: Mr. XXXXX (970) XXX-XXXX }\end{array}$ \\
\hline \multicolumn{3}{|c|}{ Mail to UNC: } \\
\hline $\begin{array}{l}\square \\
\square \\
\square \\
\square \\
\square \\
\square\end{array}$ & $\begin{array}{l}\text { 2nd signed Informed Consent Form } \\
\text { Signed HIPAA Authorization Form } \\
\text { Signed Social Security Number Disclosure Addendum } \\
\text { Copy of Laboratory Submission Form } \\
\text { Completed Practice Reimbursement Form } \\
\text { Completed Patient Reimbursement Form }\end{array}$ & $\begin{array}{l}\text { Mail to: } \\
\text { XXXXX XXXXX } \\
\text { Cecil G. Sheps Center } \\
\text { CB\#7590 } \\
\text { Chapel Hill, NC 27599-7590 }\end{array}$ \\
\hline
\end{tabular}

Before the patient leaves be sure he/she has a:

$\square$ Unsigned copy of consent form and HIPAA authorization

$\square$ Wound Care Instruction Sheet

$\square$ FOLLOW-UP APPOINTMENT

- Contact [study coordinator] for a replacement kit: (919) XXX-XXXX 


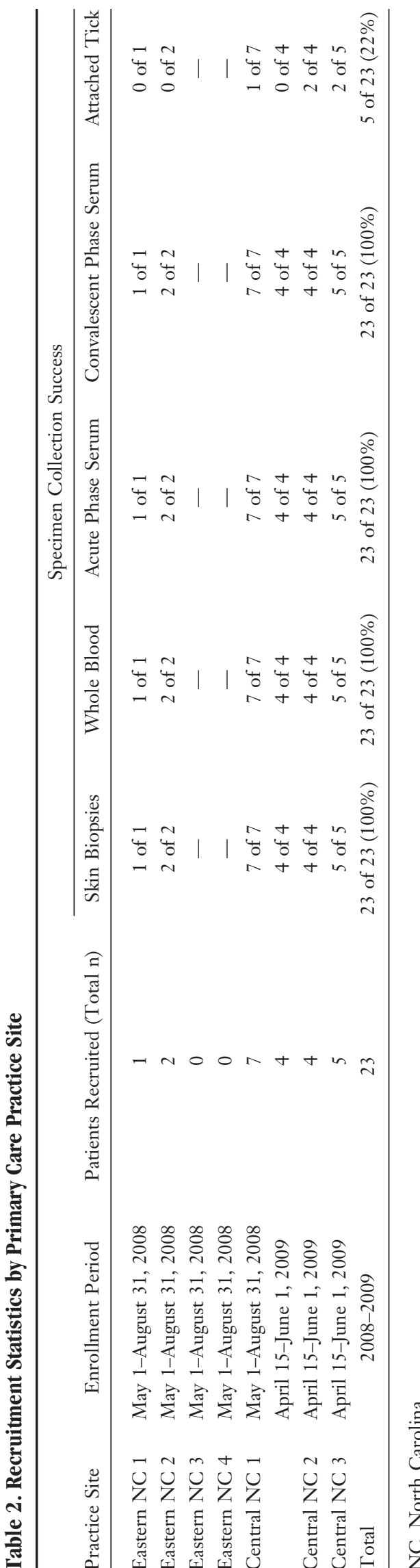

wanted more information about tick-borne diseases. Only 4 of the 28 people (14\%) who responded to the newspaper notices in 2009 were eligible to be enrolled in the study; the others did not meet eligibility criteria, with the primary reasons for noninclusion being a rash of insufficient size or previous treatment with antibiotics. All eligible patients who had telephoned were enrolled in the study. Records were not kept on the total number of calls and number of participants recruited through the newspaper notices in 2008. All patients who had been recruited through the newspaper notices were seen by one of the site coordinators and enrolled into the study within 48 hours of contacting the study coordinator.

During the 2 seasons of data collection, the study achieved $100 \%$ success in obtaining biopsy and acute and convalescent serum specimens. Recovery of tick specimens was less successful, however, with a tick being collected from only 5 of the 23 patients $(22 \%)$.

\section{Discussion}

The goal of the study was to collect biologic specimens and clinical histories from 12 to 20 patients who had STARI symptoms in North Carolina. Previous efforts to recruit and enroll STARI patients using the CDC's protocol had met with limited success. Through a collaborative partnership involving the CDC and a PBRN, recruitment and project coordination strategies were implemented that allowed the project to meet and even exceed its data collection goals. When an initial strategy was not successful, the protocol and study sites were modified to improve study yield.

Several lessons learned from this experience may be applicable to other studies aimed at recruiting patients with rare conditions from primary care offices:

1. We identified practices in high-risk areas and recruited physicians who expressed an interest in the study topic. Although a preliminary survey can be helpful in the selection of practices for participation, the investigative team must be prepared to make rapid adjustments if, for unanticipated (and in this case unclear) reasons, data collection procedures do not fulfill expectations. 
2. The study was coordinated by a universitybased study team, a process that helped assure adherence to the protocol and allowed for rapid changes in the study protocol after it did not meet with success during the initial weeks of the study.

3. Providers were offered an incentive $(\$ 1,000$ per enrollee) that was similar to that used in pharmaceutical drug trials, given the need to disrupt busy schedules and to devote time to the enrollment and data collection process. Several of the most successful providers arranged to meet study participants during off hours (eg, at 7:00 AM).

4. The continuous availability of the project coordinator to answer calls from respondents to the newspaper notices and site coordinators allowed for rapid patient assessment and visit scheduling. The site coordinator was also responsible for ensuring that all study physicians had several specimen collection kits on hand so that patients could be enrolled in the study immediately after they were identified. The timely collection of specimens had been a challenge in a previous CDC multistate protocol because specimen collection kits had to be shipped to the physician once an eligible patient was identified, and in some cases the rash would fade before a skin biopsy could be collected.

5. Weekly E-mails and use of an Internet site for communication provided a mechanism to keep physicians engaged in the study.

6. An interactive and comprehensive training session ensured that the physicians learned and understood the study protocol. The in-person, hands-on training also allowed us to engage the study physicians and give them an opportunity to ask questions and make suggestions for improvement of the protocol.

7. Additional recruitment using newspaper advertisements yielded a few cases in this project and may be useful in other studies where rare conditions are sought; however, such an approach is likely to require considerable effort to identify the minority of respondents who meet study inclusion criteria.

\section{Conclusion}

This report describes one of the first basic science research studies using a PBRN. The strategies used to address the challenges of physician and patient recruitment and complex data collection can provide guidance to investigators of future PBRNbased studies.

We would like to thank the primary care providers who enrolled patients in this study. We also thank Julia Thorp for managing patient and physician payments, and Paul Bray, MA, for assisting with project coordination in eastern North Carolina.

This research study was conducted by the North Carolina Network Consortium, a state wide collaboration of 6 PBRNs whose mission is to address pressing questions related to the delivery of primary care health services and the management of primary care problems. Participating PBRNs in this study included the North Carolina Family Medicine Research Network and the Eastern Carolina Association for Research and Education. The North Carolina Network Consortium is administered by the Cecil G. Sheps Center for Health Services Research at the University of North Carolina at Chapel Hill.

\section{References}

1. Campbell GL, Paul WS, Schriefer ME, Craven RB, Robbins KE, Dennis DT. Epidemiologic and diagnostic studies of patients with suspected early Lyme disease, Missouri, 1990-1993. J Infect Dis 1995;172: $470-80$.

2. Kirkland KB, Klimko TB, Meriwether RA, et al. Erythema migrans-like rash illness at a camp in North Carolina: a new tick-borne disease? Arch Intern Med 1997;157:2635-41.

3. Masters E, Granter S, Duray P, Cordes P. Physiciandiagnosed erythema migrans and erythema migranslike rashes following Lone Star tick bites. Arch Dermatol 1998;134:955-60.

4. Philipp MT, Masters E, Wormser GP, Hogrefe W, Martin D. Serologic evaluation of patients from Missouri with erythema migrans-like skin lesions with the C6 Lyme test. Clin Vaccine Immunol 2006;13: $1170-1$.

5. Goddard J, Varela-Stokes AS. Role of the lone star tick, Amblyomma americanum (L.), in human and animal diseases. Vet Parasitol 2009;160:1-12.

6. Masters EJ, Grigery CN, Masters RW. STARI, or Masters disease: Lone Star tick-vectored Lyme-like illness. Infect Dis Clin North Am 2008;22:361-76.

7. Paddock CD, Yabsley MJ. Ecological havoc, the rise of white-tailed deer, and the emergence of Amblyomma americanum-associated zoonoses in the United States. Curr Top Microbiol Immunol 2007;315:289-324.

8. Bacon RM, Kugeler KJ, Mead PS. Surveillance for Lyme disease-United States, 1992-2006. MMWR Surveill Summ 2008;57:1-9.

9. Wormser GP, Masters E, Liveris D, et al. Microbiologic evaluation of patients from Missouri with erythema migrans. Clin Infect Dis 2005;40:423-8.

10. Wormser GP, Masters E, Nowakowski J, et al. Pro- 
spective clinical evaluation of patients from Missouri and New York with erythema migrans-like skin lesions. Clin Infect Dis 2005;41:958-65.

11. Nicholson WL, Masters E, Wormser GP. Preliminary serologic investigation of "Rickettsia amblyommii" in the etiology of Southern tick associated rash illness (STARI). Clin Microbiol Infect 2009; 15(Suppl 2):235-6.

12. Stromdahl EY, Williamson PC, Kollars TM Jr, et al. Evidence of Borrelia lonestari DNA in Amblyomma americanum (Acari: Ixodidae) removed from humans. J Clin Microbiol 2003;41:5557-62.
13. Hummers-Pradier E, Scheidt-Nave C, Martin H, Heinemann S, Kochen MM, Himmel W. Simply no time? Barriers to GPs' participation in primary health care research. Fam Pract 2008;25:105-12.

14. Williamson MK, Pirkis J, Pfaff JJ, et al. Recruiting and retaining GPs and patients in intervention studies: the DEPS-GP project as a case study. BMC Med Res Methodol 2007;7:42.

15. Ouellette J, Apperson CS, Howard P, Evans TL, Levine JF. Tick-raccoon associations and the potential for Lyme disease spirochete transmission in the coastal plain of North Carolina. J Wildl Dis 1997; $33: 28-39$. 